\title{
Serratia liquefaciens
}

National Cancer Institute

\section{Source}

National Cancer Institute. Serratia liquefaciens. NCI Thesaurus. Code C86738.

A species of facultatively anaerobic, Gram negative, rod shaped bacteria in the phylum Proteobacteria. This species is motile, decarboxylase, citrase and esterase positive, can produce gas from glucose, and can support growth on and produce acid from arabinose, melibiose, sorbitol, and xylose. S. liquefaciens is found in water, soil and the digestive tracts of animals and has been a nosocomial pathogen for patients that received contaminated synthetic erythropoietin. 